\section{Fingers and helices}

SIR-On several occasions the interaction between Xenopus transcription factor IIIA (TFIIIA) and 5S RNA genes has been discussed in Nature, News and Views $^{1-3}$. TFIIIA is a good example of the so-called finger proteins ${ }^{4}$, eukaryotic regulatory proteins which contain variable numbers of a characteristic tendemly repeating motif of about 30 amino acids ${ }^{5,6}$. It is currently thought that these sequence repeats represent small nucleic acid binding domains, each folded about a tetrahedrally coordinated zinc ion. The coordination is provided by appropriate side chain atoms from fully conserved histidines and cysteines. This had led to a model in which TFIIIA is essentially a 'string of beads' that zigzags along one side of a nucleic acid double helix in an extended fashion?

Our observation $^{5}$ of the repeating motif in TFIIIA, which has unfortunately been overlooked by most authors, allowed us to make a reasonable prediction of the average secondary structure of the repeat. We have now extended the secondary structure analysis to include all the finger sequences known to us (Table 1). The prediction technique ${ }^{8}$ involves calculation of smoothed plots for each of the three conformational preference parameters $(\alpha$ helix, $\beta$-strand and turn) for individual sequence repeats. The smoothed curves are then averaged over all the repeats. The most strongly predicted region spans alignment positions 17-23 (Table 1) where the mean smoothed helix preference varies from 1.1 to 1.15 . Given that 1.0 indicates a neutral preference and that 1.4 is the largest preference, the helical

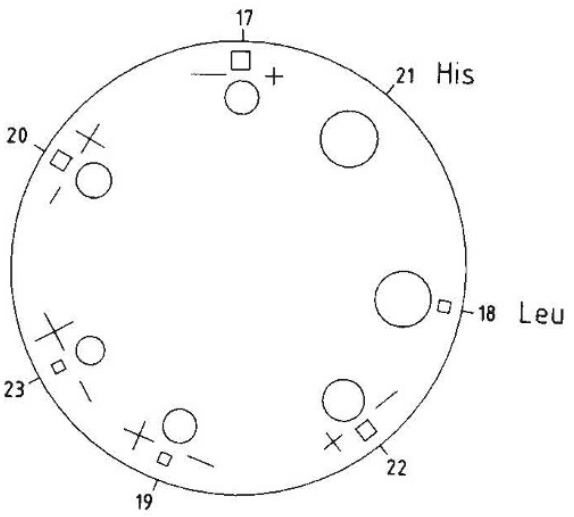

Fig. 1 A 'helical' wheel representation of the predicted $\alpha$-helical region in the 'finger' sequences. The wheel is numbered according to the alignment in Table 1 . The positions of the strongly conserved Leu 18 and His 21 are shown. The symbols used to illustrate the amphipathic nature of the $\alpha$-helix are (O) for hydrophobic residues (IALVMCHYWF), $(+)$ for basic amino acids (KR), (-) for acidic/polar residues (QEDN), and (ם) for (PSTG). The size of the symbols are proportional to the amino acid composition at each alignment position.

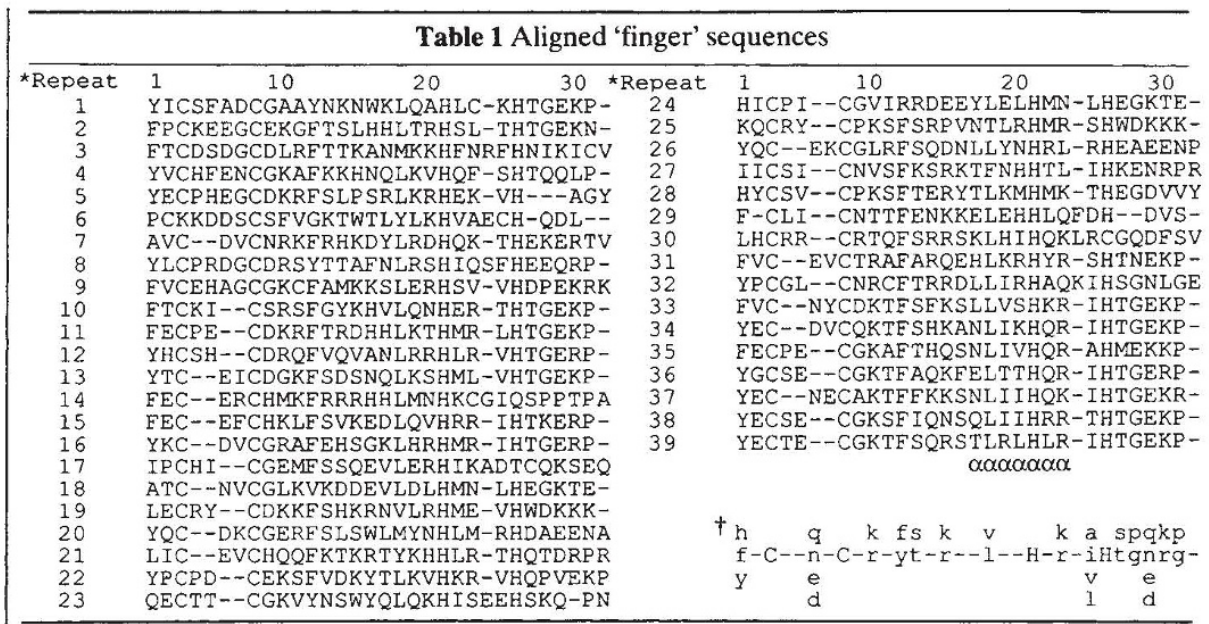

${ }^{*}$ Repeats $1-9$ are from Xenopus TFIIIA. $10-14$ are from Drosophila $K t, 15-16$ from $K r h$ (R. Schuh and H. Käckle), $17-22$ from beta sry, $23-29$ from detla sry and 30 from IB142 (R. Baldarelli and J. Lengvel). $31-32$ are from yeast ADRI and $33-39$ from mouse MKr3 (K. Chowdhury and P. Gruss)

$\dagger$ The consensus for an alignment position is given if 19 or more of the 39 residues are conserved according to the groups: (ST), (PG), (KR), (QNED), (MC), (HFYW) and (AIVL).

prediction over the 39 repeats is clear.

An $\alpha$-helical wheel representation ${ }^{9}$ of the span (Fig. 1) shows an obvious hydrophobic/hydrophilic sidedness, typical of helices observed in many threedimensional protein structures. One of the conserved histidines suggested for zinc coordination and a strongly conserved leucine are located on the hydrophobic side of the predicted $\alpha$-helix. This span may act as the principal finger region for interaction with nucleic acid.

Two preliminary spectroscopic observations in this laboratory support the helical suggestion. Circular dichroism curves of the TFIIIA protein as analysed by the method of Provencher ${ }^{10}$ indicate the presence of $10-15$ per cent $\alpha$-helix. A fluorescence quenching analysis of the $7 \mathrm{~S}$ particle shows an approximate 30 per cent increase in intensity upon digestion of the RNA with ribonucleases or its saltinduced dissociation. We interpret this re-

\section{Data in graphs and tables}

SIR-After reading Paolini's discussion on enzyme units and how to express them ${ }^{1}$, my attention was drawn to a similar ambiguity in the literature, concerning the units in which data are expressed when shown in tables or plotted in graphical form.

Basic mathematics tells us that the value of a physical quantity is equal to the product of a numerical value and a unit:

physical quantity $=$ numerical value $\times$ unit

According to the convention followed by the Royal Society ${ }^{2}$, the expression used to define the numerical values of a physical quantity plotted on a graph (or the one which is placed at the head of a column of numerical values of a physical quantity in sult as evidence for changes in the environments of the two tryptophan residues (W28 and W177) in TFIIIA which are just at the amino-terminal side of the predicted $\alpha$-helix. Apparently their degree of exposure and structural flexibility is altered considerably upon binding to nucleic acid.

RAYMOND S. BROWN PATRICK Argos

European Molecular Biology Laboratory, Meyerhofstrasse 1,

\section{D-6900 Heidelberg, FRG}

1. Enver, 'T. Nature 317, 385 - 386 (1985).

2. Berg, J.M. Nature 319, $264-265$ (1986).

3. Harrison, S.C. Nature $322,597-598(1986)$

4. Vincent, A. Nucleic Acid Res. 14, 4385 - 4391 (1986)

5rown, R.S., Sander, C. \& Argos, P. FEBS LETT. 186, $271-274(1985)$

6. Miller, J., McLachlan, A.D. \& Klug, A. EMBO J. 4, 1609 - 1614 (1985)

7. Rhodes, D. \& Klug, A. Cell 46, 123 - 132 (1986)

8. Argos, P. EMBO J. 4, $1351-1355$ (1985)

9. Schiffer, M. \& Edmundson, A.B. Biophys. J. 7, 121 - 135 (1967)

10. Provencher, S.W. \& Glöckner, J. Biochemistry 20, 33 - 37 $(1981)$

a table) should be a pure number, and the axis on the graph (or the head of the table) should be labelled with physical quantity/ unit - for example, radioactivity/(d.p.m. $\left.\times 10^{3}\right)-$ thus, making the numbers in graphs and tables dimensionless. If this convention is not followed, that is, if the numerical values in graphs and tables are considered to be dimensional, then the axis on graphs and the heads of tables should be labelled with the appropriate units (d.p.m. $\times 10^{3}$, and not d.p.m. $\times 10^{-3}$ as is often the case).

Figure 1 summarizes several ways of expressing data in graphical form. In this figure, molar was the chosen unit because conventional prefixes like nano, micro, milli, and so on, are very commonly used, which is not the case with d.p.m. (for which $\mathrm{Bq}$ is now the recommended unit) 